\title{
Letter
}

\section{Central Research Labs In Medical Colleges}

\author{
Nitin Ashok John
}

\section{To the editor}

The Central Research Laboratory is built to provide teaching staff, researchers and graduate students with easy access to costly research equipment. Central Research Labs should be equipped with sophisticated equipments such as electron microscopes, nucleic acid structure analysis systems, image analysis processors and mass spectrometers under a centralized control system. For the further development of modern medicine, it is indispensable to be fully equipped with advanced technologies such as medical, analytical and information devices, and to utilize them in a systematic manner.

- It is also essential to promote academic exchanges on a global basis to provide tangible and intangible support.

- Installation of various devices, such as genetic research equipment and an electron spin resonance apparatus, for establishing leading-edge scientific and research including that on vascularization, vascular remodeling and apoptosis, endothelial and free radical injuries and vascular tonus can be carried out under interdisciplinary team.

- To effectuate appropriate ethical and scientific practice in animal experiments, guidance and lectures should be regularly given on experimental guidelines including the handling of animals.

- The Biomedical Computation Center should be built to achieve effective utilization of the "distributed artificial intelligence system". A high-speed graphic workstation and a highspeed statistics data processing workstation should be introduced for providing valuable information to teaching staffs.

- Department of Biochemistry has very special role to play and should effectively support the analytical phase of clinical trials in many different areas in a wide range of matrices including plasma, serum, urine, CSF and whole blood. Central Research Laboratory should

Received April 2011; Accepted November 2011 be able to offer a flexible service that supports each unique study.

- Facilities that can be incorporated are laboratory services exclusively for clinical trials, bioanalytical/bio equivalence and research cohort studies, clinical assays with on-line data capture, specimen management, sample tracking and storage systems, extensive in-house specialty testing and esoteric capability, flexible data management and rapid results reporting and dedicated project management and client support services

- The team of specialists from all medical specialties should included in the Central Research Lab Monitoring Committee.the other services which can be catered from the central research lab are providing guidelines for economic modeling, prospective study design, execution and analysis, disease management design, execution and evaluation, psychometric development and validation, commercial reimbursement and formulator placement and policy analytics and legislativ briefings.

Effective management

- A highly dedicated and devoted Research Unit Head with academically extra ordinary achievers from all specialties as members.

- All faculty members should strive for getting funding from external agencies.

- A good statistical unit should be attached to the lab for efficient guideline to the researchers.

- A publication Cell with team members who will assist, review and help the faculty members in publishing the scientific work.

*Nitin Ashok John MD, Professor and Head, Department Physiology, Indira Gandhi Medical College and Research Institute, Government of Pondicherry, India, 99, Khare Tarakunde Nagar, Katol Road, Nagpur 440013. India, e-mail:drnitinjohn@yahoo.co.in

* for correspondance 